\title{
Goldstern-Judah-Shelah preservation theorem for countable support iterations
}

\author{
by
}

\author{
Miroslav Re pi c ḱ́ (Košice)
}

\begin{abstract}
In [4] a preservation theorem for countable support iterated forcing is proved with restriction to forcing notions which are not $\omega$-distributive. We give the proof of the theorem without this restriction.
\end{abstract}

1. The preservation theorem. In [4] a preservation theorem for countable support iteration of proper forcing notions was proved with the additional assumption that all forcing notions which are iterated add a new sequence of ordinals. In this section we will prove the same theorem (Theorem 1.7) without this additional assumption. We use the terminology introduced in [4]; definitions and lemmata 1.1-1.8 correspond to 5.4, 5.5, 5.6, $5.8,5.11,5.12,5.14,5.13$ of [4]. Lemma 1.9 is a version of 5.15 without the additional assumption and essentially it marks the difference between these two proofs of the preservation theorem.

Let $\left\langle\sqsubseteq_{n}: n \in \omega\right\rangle$ be an increasing sequence of two-place relations on ${ }^{\omega} \omega$. We let $\sqsubseteq=\bigcup_{n} \sqsubseteq_{n}$. We assume the following:

(i) $\left\{f \in{ }^{\omega} \omega: f \sqsubseteq_{n} g\right\}$ is a closed set for any $n \in \omega$ and $g \in{ }^{\omega} \omega$;

(ii) the set $C=\operatorname{dom}(\sqsubseteq)$ is a closed subset of ${ }^{\omega} \omega$;

(iii) for every countable set $A \subseteq C$ there is $g \in{ }^{\omega} \omega$ such that $\forall f \in A$, $f \sqsubseteq g$;

(iv) the closed sets mentioned in conditions (i) and (ii) have an absolute definition (i.e. as Borel sets they have the same Borel codes in all transitive models we will consider).

1991 Mathematics Subject Classification: Primary 03E40.

Key words and phrases: countable support iterated forcing, proper forcing, preservation theorem for iterated forcing.

The work was partially supported by BRF of Israel Academy of Sciences and by grant GA SAV 365/92 of Slovak Academy of Sciences. 
In all our applications the relations $\sqsubseteq_{n}$ will be even given by arithmetical definitions, and so they will be absolute between any two $\in$-models.

For rng $(\sqsubseteq)$ we do not need any assumption analogous to condition (ii) for dom( $\sqsubseteq)$. Even, rng( $\sqsubseteq$ ) can be an arbitrary set (not necessarily a set of reals) which is assumed not to change during the iteration. So the preservation theorem says that although in $\operatorname{dom}(\sqsubseteq)$ new reals appear, some relations between (new) domain and (old) range of $\sqsubseteq$ are preserved.

The letter $C$ will always denote $\operatorname{dom}(\sqsubseteq)$. We will use the symbol $\sqsubseteq$ also for the sequence $\left\langle\Xi_{n}: n \in \omega\right\rangle$.

Definition 1.1. Let $N$ be a countable elementary substructure of some $H(\chi)$ such that $\sqsubseteq \in N$. We say that $g$ covers $N$ if for all $f \in C \cap N$ we have $f \sqsubseteq g$.

Definition 1.2. We say that a forcing notion $P$ almost preserves $\sqsubseteq$ if whenever $N \prec H(\chi)$ is a countable substructure containing $P$, 5 and whenever $p \in P \cap N$ and $g$ covers $N$ then there is an $N$-generic condition $q \leq p$ such that $q \Vdash$ " $g$ covers $N[\dot{G}]$ ".

Lemma 1.3. Let $P$ be a proper forcing notion. If $P$ almost preserves $\sqsubseteq$ then $\Vdash_{P}(\forall f \in C \cap V[\dot{G}])(\exists g \in V) f \sqsubseteq g$. Moreover, if $\sqsubseteq$ is transitive then these two properties are equivalent.

Proof. Assume there is a condition $p$ and a name $\dot{f}$ such that $p \Vdash$ "there is no $g \in V$ such that $\dot{f} \sqsubseteq g$ ". Let $N \prec H(\chi)$ and let $\dot{f}, p \in N$. There is $g \in V$ which covers $N$. Since $P$ almost preserves $\sqsubseteq$ there is $q \leq p$ such that $q \Vdash \dot{f} \sqsubseteq g$, a contradiction.

Conversely, let $N \prec H(\chi)$ and assume that $g$ covers $N$ and

$$
\Vdash_{P}(\forall f \in C)\left(\exists f^{\prime} \in V\right) f \sqsubseteq f^{\prime} .
$$

Then any $N$-generic condition $q$ forces: $q \Vdash(\forall f \in C \cap N[\dot{G}])\left(\exists f^{\prime} \in N\right) f \sqsubseteq f^{\prime}$. Hence, by transitivity of $\sqsubseteq$ we get $q \Vdash(\forall f \in C \cap N[\dot{G}]) f \sqsubseteq g$.

We would like to preserve the properties mentioned in Lemma 1.3 in countable support iterations. However, for our preservation theorem we need a slightly stronger property (Definition 1.5). Fortunately, in some interesting cases these three properties coincide.

Before we give the definition which we will actually use we have to recall the concept of interpretation.

Definition 1.4. Assume $P$ is a forcing notion, $\dot{f}_{0}, \ldots, \dot{f}_{k}$ are $P$-names of functions in $C, f_{0}^{*}, \ldots, f_{k}^{*}$ are functions in ${ }^{\omega} \omega$ and $\left\langle p_{n}: n \in \omega\right\rangle$ is a decreasing sequence of conditions. We say that $\left\langle p_{n}: n \in \omega\right\rangle$ interprets $\left\langle\dot{f}_{0}, \ldots, \dot{f}_{k}\right\rangle$ as $\left\langle f_{0}^{*}, \ldots, f_{k}^{*}\right\rangle$ if for all $i \leq k$ and for all $n, p_{n} \Vdash \dot{f}_{i}\left\lceil n=f_{i}^{*}\lceil n\right.$. 
Note that if $\left\langle p_{n}: n \in \omega\right\rangle$ interprets $\dot{f}$ as $f^{*}$ where $\dot{f}$ is a $P$-name for a function in $C$ then $f^{*}$ is a function in $C$. This is because $C$ is closed.

Definition 1.5. We say that a forcing notion $P$ preserves $\sqsubseteq$ if: whenever $N \prec H(\chi)$ is a countable model containing $P$ and $\sqsubseteq$ and whenever $g$ covers $N$ and $\left\langle p_{n}: n \in \omega\right\rangle$ in $N$ is a decreasing sequence of conditions interpreting $\left\langle\dot{f}_{0}, \ldots, \dot{f}_{k}\right\rangle \in N$ as $\left\langle f_{0}^{*}, \ldots, f_{k}^{*}\right\rangle$ then there is an $N$-generic condition $p \leq p_{0}$ such that

(a) $p \Vdash ~ " g$ covers $N[\dot{G}]$ ", and

(b) $(\forall n \in \omega)(\forall i \leq k) p \Vdash f_{i}^{*} \sqsubseteq_{n} g \rightarrow \dot{f}_{i} \sqsubseteq_{n} g$.

The following lemma is a special case of the preservation theorem we are going to prove. Although we will not use this result in the proof of the preservation theorem, an easy trick used in the proof will be repeated later in a somewhat more complicated situation.

Lemma 1.6. If $P$ preserves $\sqsubseteq$ and $\Vdash_{P}$ " $\dot{Q}$ preserves $\sqsubseteq$ " then $P * \dot{Q}$ preserves $\sqsubseteq$.

Proof. Assume $N,\left\langle\left\langle p_{n}, \dot{q}_{n}\right\rangle: n \in \omega\right\rangle,\left\langle\dot{f}_{0}, \ldots, \dot{f}_{k}\right\rangle,\left\langle f_{0}^{*}, \ldots, f_{k}^{*}\right\rangle$ are as in Definition 1.5 (for the forcing notion $P * \dot{Q} \in N$ with $\left\langle p_{n}, \dot{q}_{n}\right\rangle$ in the role of $p_{n}$ ) and let $g$ cover $N$.

In $V^{P}$ we define names of functions $\dot{f}_{i}^{\prime}$ for $i \leq k$ and names for conditions $\dot{q}_{n}^{\prime} \in \dot{Q}$ as follows: Let $\dot{n}^{*}=\sup \left\{n: p_{n} \in \dot{G}\right\}$. If $\dot{n}^{*}=\omega$ then set $\dot{f}_{i}^{\prime}=f_{i}^{*}$ and $\dot{q}_{n}^{\prime}=\dot{q}_{n}$. If $\dot{n}^{*}<\omega$ then find a decreasing sequence $\left\langle\dot{q}_{n}^{\prime}: n \in \omega\right\rangle \in{ }^{\omega} \dot{Q}$ and functions $\dot{f}_{i}^{\prime}, i \leq k$, such that $\dot{q}_{0}^{\prime} \leq \dot{q}_{\dot{n}^{*}}$ and $\left\langle\dot{q}_{n}^{\prime}: n \in \omega\right\rangle$ interprets $\left\langle\dot{f}_{0}, \ldots, \dot{f}_{k}\right\rangle$ as $\left\langle\dot{f}_{0}^{\prime}, \ldots, \dot{f}_{k}^{\prime}\right\rangle$ in $V^{P}$.

Observe that $p_{0} \Vdash_{P} \dot{f}_{i}^{\prime}\left\lceil\dot{n}^{*}=f_{i}^{*}\left\lceil\dot{n}^{*}\right.\right.$ and $p_{n} \Vdash_{P} \dot{n}^{*} \geq n$. In particular, $p_{n} \Vdash_{P} \dot{f}_{i}^{\prime} \uparrow n=f_{i}^{*}\lceil n$.

We can find all these names in $N$. Since $P$ preserves $\sqsubseteq$, there is an $N$-generic condition $p \in P, p \leq p_{0}$, such that $p \Vdash$ " $g$ covers $N\left[\dot{G}_{P}\right]$ " and for $n \in \omega$ and $i \leq k$, if $\dot{f}_{i}^{*} \sqsubseteq_{n} g$ then $p \Vdash_{P} \dot{f}_{i}^{\prime} \sqsubseteq_{n} g$. Now using the preserving property of $\dot{Q}$ we can find in $V^{P}$ a name $\dot{q} \in \dot{Q}$ of an $N\left[\dot{G}_{P}\right]$-generic condition such that $\dot{q} \leq \dot{q}_{0}^{\prime}$ and such that

$$
p \Vdash_{P} \dot{q} \Vdash_{\dot{Q}} \text { " } g \text { covers } N\left[\dot{G}_{P} * \dot{G}_{\dot{Q}}\right] \text { and if } f_{i}^{\prime} \sqsubseteq_{n} g \text { then } \dot{f}_{i} \sqsubseteq_{n} g \text { ". }
$$

It can easily be seen that the condition $(p, \dot{q})$ has the required properties.

THEOREM 1.7. If $\left\langle P_{\alpha}, \dot{Q}_{\alpha}: \alpha<\delta\right\rangle$ is a countable support iterated forcing system and for each $\alpha<\delta, \vdash_{\alpha}$ " $\dot{Q}_{\alpha}$ preserves $\sqsubseteq$ " then $P_{\delta}$ preserves $\sqsubseteq$.

Proof. Let $N \prec H(\chi)$ be a countable elementary substructure containing the forcing system $\left\langle P_{\alpha}, \dot{Q}_{\alpha}: \alpha<\delta\right\rangle$ and $P_{\delta}$. We shall show by induction on $\beta \leq \delta$ that every $P_{\beta} \in N$ has a somewhat stronger property than Definition 1.5 requires. 
Recall that for $\alpha<\beta, \dot{P}_{\alpha, \beta}$ is a $P_{\alpha}$-name for a forcing notion with the property that $P_{\alpha} * \dot{P}_{\alpha, \beta}$ can be densely embedded into r.o. $P_{\beta}$. We define it so that the domain of this name is formed by functions $p \in P_{\beta}$ with $p\left\lceil\alpha=\mathbf{1}_{P_{\alpha}}\right.$ and for two such functions $p, p^{\prime}, \Vdash_{\alpha} p \leq_{\alpha, \beta} p^{\prime}$ iff $p \leq_{\beta} p^{\prime}$.

Lemma 1.8. Let $\alpha<\beta \leq \delta, \alpha, \beta \in N$. Assume that $\left\langle\dot{p}_{n}: n \in \omega\right\rangle \in N$ is a sequence of $P_{\alpha}$-names for conditions in $\dot{P}_{\alpha, \beta}$ such that $\Vdash_{\alpha}$ " $\left\langle\dot{p}_{n}: n \in \omega\right\rangle$ interprets $\left\langle\dot{f}_{0}, \ldots, \dot{f}_{k}\right\rangle$ as $\left\langle\dot{f}_{0}^{*}, \ldots, \dot{f}_{k}^{*}\right\rangle$ ", where the $\dot{f}_{i}$ are $P_{\beta}$-names in $N$ and the $\dot{f}_{i}^{*}$ are $P_{\alpha}$-names, for $i \leq k$. Furthermore, assume $p \in P_{\alpha}$ is $N$-generic and for some $g, p \Vdash_{\alpha}$ " $g$ covers $N\left[\dot{G}_{\alpha}\right]$ ". Then there exists $r \in P_{\beta}$, an $N$ generic condition, such that $r\lceil\alpha=p$ and

(a) $r \Vdash_{\beta}$ "g covers $N\left[\dot{G}_{\beta}\right]$ ";

(b) $r \Vdash_{\beta} \dot{f}_{i}^{*} \sqsubseteq_{n} g \rightarrow \dot{f}_{i} \sqsubseteq_{n} g$, for $n \in \omega, i \leq k$;

(c) $p \Vdash_{\alpha} r \uparrow\langle\alpha, \beta) \leq \dot{p}_{0}$.

In the proof of the case " $\beta$ limit" of Lemma 1.8 we will need the following lemma.

Lemma 1.9. Assume $0<\alpha^{\prime}<\beta$, $\beta$ limit, $\left\langle p_{n}: n \in \omega\right\rangle \in N$ is a decreasing sequence of conditions in $P_{\beta}$. Let $\tau \in N$ be a $P_{\beta}$-name of an ordinal and $j \in \omega$. Assume $\left\langle\dot{f}_{0}, \ldots, \dot{f}_{k+1}\right\rangle \in N$ are $P_{\beta}$-names of functions and $\left\langle p_{n}: n \in \omega\right\rangle$ interprets $\left\langle\dot{f}_{0}, \ldots, \dot{f}_{k}\right\rangle$ as $\left\langle f_{0}^{*}, \ldots, f_{k}^{*}\right\rangle$. Then there are $\alpha \in N$, with $\alpha^{\prime} \leq \alpha<\beta$, a $P_{\alpha}$-name for a decreasing sequence $\left\langle p_{n}^{*}: n \in \omega\right\rangle$ of conditions in $\dot{P}_{\alpha, \beta}, P_{\alpha}$-names $\left\langle\dot{f}_{0}^{\prime}, \ldots, \dot{f}_{k+1}^{\prime}\right\rangle \in N$ and a $P_{\alpha}$-name for $a$ sequence of conditions in $\dot{P}_{\alpha, \beta},\left\langle\dot{p}_{n}^{\prime}: n \in \omega\right\rangle \in N$ such that

(a) $\Vdash_{\alpha}$ “ $\left\langle\dot{p}_{n}^{\prime}: n \in \omega\right\rangle$ interprets $\left\langle\dot{f}_{0}, \ldots, \dot{f}_{k+1}\right\rangle$ as $\left\langle\dot{f}_{0}^{\prime}, \ldots, \dot{f}_{k+1}^{\prime}\right\rangle "$;

(b) $\Vdash_{\alpha}$ " $\dot{p}_{0}^{\prime}$ decides $\dot{f}_{0} \nmid j, \ldots, \dot{f}_{k+1}\lceil j "$;

(c) $\Vdash_{\alpha} p_{0}^{\prime} \Vdash_{\alpha, \beta} \tau \in N\left[\dot{G}_{\alpha}\right]$;

(d) $p_{n}^{*}\left\lceil\alpha \Vdash_{\alpha} \dot{p}_{0}^{\prime} \leq p_{n}^{*}\lceil\langle\alpha, \beta)\right.$;

(e) $\left\langle p_{n}^{*}\lceil\alpha: n \in \omega\rangle\right.$ interprets $\left\langle\dot{f}_{0}^{\prime}, \ldots, \dot{f}_{k}^{\prime}\right\rangle$ as $\left\langle f_{0}^{*}, \ldots, f_{k}^{*}\right\rangle$;

(f) $p_{n}^{*} \leq p_{n}$ for $n \in \omega$ (and in fact $p_{n}^{*} \uparrow\langle\alpha, \beta)=p_{n} \uparrow\langle\alpha, \beta)$ ).

Proof. We say that a sequence $\left\langle p_{n}: n \in \omega\right\rangle$ of conditions in $P_{\beta}$ is consistent if it has a lower bound, otherwise it is inconsistent. We say that it is strongly inconsistent if $\min \left\{\xi:\left\{p_{n} \mid \xi: n \in \omega\right\}\right.$ is inconsistent $\}<\beta$.

(i) First assume that $\left\langle p_{n}: n \in \omega\right\rangle$ is consistent. Let $\alpha=\alpha^{\prime}, p_{n}^{*}=p_{n}$ and in $V^{P_{\alpha}}$ define $\dot{n}^{*}=\sup \left\{n: p_{n}^{*}\left\lceil\alpha \in \dot{G}_{\alpha}\right\}\right.$ and $\dot{p}_{n}^{\prime}, \dot{f}_{i}^{\prime}$ so that

(1) if $\dot{n}^{*}<\omega$ then $\dot{p}_{0}^{\prime} \leq p_{\dot{n}^{*}} \uparrow\langle\alpha, \beta)$;

(2) if $\dot{n}^{*}=\omega$ then $\dot{p}_{0}^{\prime} \leq p_{n} \uparrow\langle\alpha, \beta)$, for all $n \in \omega$;

(3) if $\dot{n}^{*}$ is not defined then $\dot{p}_{0}$ is arbitrary,

but in all these cases $\dot{p}_{n}^{\prime}$ is chosen so that $\dot{p}_{0}^{\prime}$ decides $\tau, \dot{f}_{0}\left\lceil j, \ldots, \dot{f}_{k+1}\lceil j\right.$, and $\left\langle\dot{p}_{n}^{\prime}: n \in \omega\right\rangle$ interprets $\left\langle\dot{f}_{0}, \ldots, \dot{f}_{k+1}\right\rangle$ as $\left\langle\dot{f}_{0}^{\prime}, \ldots, \dot{f}_{k+1}^{\prime}\right\rangle$ in $V^{P_{\alpha}}$. 
Clearly all these names can be found in $N$ and also conditions (a), (b), (f) are satisfied. To see (c) it is enough to realize that the name $\tau$ is forced by conditions in $N$ so its decision is forced to be in $N\left[\dot{G}_{\alpha}\right]$.

(d) By the construction, $p_{n}^{*}\left\lceil\alpha \Vdash_{\alpha}\right.$ " $\dot{n}^{*} \geq n$ ". Hence $p_{n}^{*}\left\lceil\alpha \Vdash_{\alpha}\right.$ "if $\dot{n}^{*}<\omega$, $\dot{p}_{0}^{\prime} \leq p_{\dot{n}^{*}}^{*} \uparrow\langle\alpha, \beta) \leq p_{n}^{*} \uparrow\langle\alpha, \beta)$ " and also by the definition $p_{n}^{*}\left\lceil\alpha \Vdash_{\alpha}\right.$ "if $\dot{n}^{*}=\omega$ then $\dot{p}_{0}^{\prime} \leq p_{n}^{*} \uparrow\langle\alpha, \beta)^{\prime \prime}$.

(e) By (d), $p_{n}^{*}\left\lceil\alpha \Vdash_{\alpha} \dot{p}_{n}^{\prime} \leq p_{n}^{*}\left\lceil\langle\alpha, \beta)\right.\right.$, hence $p_{n}^{*}\left\lceil\alpha \Vdash_{\alpha} \dot{p}_{n}^{\prime} \Vdash_{\alpha, \beta} \dot{f}_{i}^{\prime}\lceil n=\right.$ $\dot{f}_{i} \uparrow n=f_{i}^{*}\left\lceil n, i \leq k\right.$. But the formula " $\dot{f}_{i}^{\prime} \uparrow n=f^{*}\lceil n$ " is an (absolute) formula about $V^{P_{\alpha}}$, so $p_{n}^{*}\left\lceil\alpha \Vdash_{\alpha} \dot{f}_{i}^{\prime}\left\lceil n=f_{i}^{*}\lceil n\right.\right.$.

(ii) Assume that $\left\langle p_{n}: n \in \omega\right\rangle$ is strongly inconsistent. Let $\alpha=\min \{\xi \geq$ $\alpha:\left\langle p_{n} \mid \xi: n \in \omega\right\rangle$ is inconsistent $\}<\beta, p_{n}^{*}=p_{n}$ and in $V^{P_{\alpha}}$ define $\dot{n}^{*}=$ $\sup \left\{n: p_{n}^{*}\left\{\alpha \in G_{\alpha}\right\}<\omega\right.$ and $p_{n}^{\prime}, \dot{f}_{i}^{\prime}$ so that (1) and (3) hold. Then the proof that (a)-(f) are satisfied is the same as in case (i).

(iii) Finally, assume that $\left\langle p_{n}: n \in \omega\right\rangle$ is inconsistent but not strongly inconsistent. We work in $N$.

We claim that there is $\gamma, \alpha^{\prime} \leq \gamma<\beta$, and $q \in P_{\gamma}$ such that $q \leq p_{n}\lceil\gamma$, for all $n$, and $q \Vdash_{\gamma}$ " $\left\langle p_{n}\lceil\langle\gamma, \beta): n \in \omega\rangle\right.$ is strongly inconsistent".

Assume that the claim is not true. Let $\xi_{n}, n \in \omega$, be an increasing sequence of ordinals with $\xi_{0}=0$ and $\lim _{n} \xi_{n}=\sup (N \cap \beta)$. By induction on $k \in \omega$ we can construct $P_{\xi_{k}}$-names $\dot{q}_{k} \in \dot{P}_{\xi_{k}, \xi_{k+1}}$ such that $\dot{q}_{k} \leq p_{n} \uparrow\left\langle\xi_{k}, \xi_{k+1}\right)$ for all $n \in \omega$ (forced in $\left.P_{\xi_{k}}\right)$ and $\left(\dot{q}_{0}, \ldots, \dot{q}_{k}\right) \Vdash_{\xi_{k+1}} "\left\langle p_{n} \mid \xi_{k+1}: n \in \omega\right\rangle$ is not strongly inconsistent".

But since we deal with proper forcing notions this leads to a construction of a lower bound for $\left\langle p_{n}: n \in \omega\right\rangle$. A contradiction.

Assume $q, \gamma$ satisfy the above claim. Then for some extension $q^{\prime} \leq_{\gamma} q$ and for some $\gamma^{\prime}$ with $\gamma<\gamma^{\prime}<\beta, q^{\prime} \Vdash_{\gamma}$ " $\left\langle p_{n} \mid\left\langle\gamma, \gamma^{\prime}\right): n \in \omega\right\rangle$ is inconsistent". So the sequence $p_{n}^{*}=q^{\prime} \cup p_{n}\lceil\langle\gamma, \beta)$ is strongly inconsistent and we proceed with it exactly as in case (ii) (with a strongly inconsistent sequence $\left\langle p_{n}: n \in \omega\right\rangle$ ).

Proof of Lemma 1.8. By induction on $\beta \leq \delta, \beta \in N$. For $\beta=0$ the lemma is trivially true. Now assume that the lemma holds for $\beta$ and we prove it for $\beta+1$.

Let $\alpha<\beta+1$. Assume that $\left\langle\dot{p}_{n}: n \in \omega\right\rangle \in N$ is a $P_{\alpha}$-name for a decreasing sequence in $\dot{P}_{\alpha, \beta+1}$ which in $V^{P_{\alpha}}$ interprets $\left\langle\dot{f}_{0}, \ldots, \dot{f}_{k}\right\rangle$ as $\left\langle\dot{f}_{0}^{*}, \ldots, \dot{f}_{k}^{*}\right\rangle$.

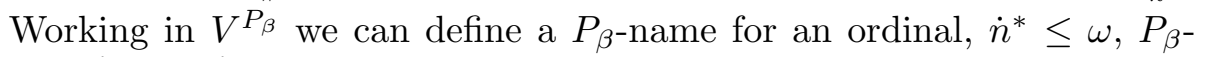
names $\left\langle\dot{f}_{0}^{\prime}, \ldots, \dot{f}_{k}^{\prime}\right\rangle$ for functions in ${ }^{\omega} \omega$, and a $P_{\beta}$-name $\left\langle\dot{p}_{n}^{\prime}: n \in \omega\right\rangle$ for a decreasing sequence in $\dot{Q}_{\beta}$ such that

(1) $\dot{n}^{*}=\sup \left\{n \in \omega: \dot{p}_{n}\left\lceil\beta \in \dot{G}_{\beta}\right\}\right.$;

(2) if $\dot{n}^{*}<\omega$ then $\dot{p}_{0}^{\prime} \leq \dot{p}_{\dot{n}^{*}}(\beta)$; and if $\dot{n}^{*}=\omega$ then $\dot{p}_{n}^{\prime}=\dot{p}_{n}(\beta)$ and $\left\langle\dot{f}_{0}^{\prime}, \ldots, \dot{f}_{k}^{\prime}\right\rangle=\left\langle\dot{f}_{0}^{*}, \ldots, \dot{f}_{k}^{*}\right\rangle$ 
(3) in the case of $\dot{n}^{*}<\omega,\left\langle\dot{p}_{n}^{\prime}: n \in \omega\right\rangle$ is such that $\dot{p}_{n}^{\prime}$ decides $\dot{f}_{i}\lceil n$ and $\dot{p}_{n}^{\prime} \Vdash_{\dot{Q}_{\beta}} \dot{f}_{i} \uparrow n=\dot{f}_{i}^{\prime}\lceil n, i \leq k$.

As in the proof of Lemma 1.6,

(4) $\Vdash_{\alpha}$ " $\left\langle\dot{p}_{n}\lceil\beta: n \in \omega\rangle\right.$ interprets $\left\langle\dot{f}_{0}^{\prime}, \ldots, \dot{f}_{k}^{\prime}\right\rangle$ as $\left\langle\dot{f}_{0}^{*}, \ldots, \dot{f}_{k}^{*}\right\rangle "$, and

$(5) \Vdash_{\alpha}$ " $\dot{p}_{0}\left\lceil\beta \Vdash_{\alpha, \beta}\left\langle\dot{p}_{n}^{\prime}: n \in \omega\right\rangle\right.$ interprets $\left\langle\dot{f}_{0}, \ldots, \dot{f}_{k}\right\rangle$ as $\left\langle\dot{f}_{0}^{\prime}, \ldots, \dot{f}_{k}^{\prime}\right\rangle "$.

Now using the induction hypothesis for $\beta$, there is an $N$-generic condition $r \in P_{\beta}$ such that $r\lceil\alpha=p$ and

(6) $r \Vdash_{\beta}$ "g covers $N\left[\dot{G}_{\beta}\right]$ ";

(7) $r \Vdash_{\beta}(\forall n \in \omega)(\forall i \leq k) \dot{f}_{i}^{*} \sqsubseteq_{n} g \rightarrow \dot{f}_{i}^{\prime} \sqsubseteq_{n} g$;

(8) $p \Vdash_{\alpha} r \uparrow\langle\alpha, \beta) \leq \dot{p}_{0}\lceil\beta$.

Hence using the hypothesis on $\dot{Q}_{\beta}$, since (5) holds, we can find a $P_{\beta}$-name $\dot{s}$ for an $N\left[\dot{G}_{\beta}\right]$-generic condition in $\dot{Q}_{\beta}$ such that

(9) $r \Vdash_{\beta}$ "s $s \dot{p}_{0}^{\prime} \leq \dot{p}_{0}(\beta) \& \dot{s} \Vdash_{\dot{Q}_{\alpha}} g$ covers $N\left[\dot{G}_{\beta}\right]\left[\dot{G}_{\dot{Q}_{\beta}}\right]$ ", and

$(10) \Vdash_{\beta} \dot{s} \Vdash_{\dot{Q}_{\beta}}(\forall n \in \omega)(\forall i \leq k) \dot{f}_{n}^{\prime} \sqsubseteq_{n} g \rightarrow \dot{f}_{i} \sqsubseteq_{n} g$.

So (7)-(10) say that the condition $(r, \dot{s}) \in P_{\beta+1}$ is as required and the successor step of the induction is finished.

Now let $\beta \in N$ be a limit ordinal, $\beta \leq \delta$. Let $\left\{\tau_{n}: n \in \omega\right\}$ be an enumeration of all $P_{\beta}$-names of ordinals in $N$, and $\left\{\dot{f}_{n}: n \in \omega\right\}$ be an enumeration of all $P_{\beta}$-names $\dot{f} \in N$ satisfying $\Vdash_{\beta} \dot{f} \in C$ with the first $k+1$ members already fixed. Let $\alpha<\beta, \alpha \in N$.

So $p \in P_{\alpha}$ is an $N$-generic condition, $p \Vdash_{\alpha}$ " $g$ covers $N\left[\dot{G}_{\alpha}\right]$ ", and $\Vdash_{\alpha}$ " $\left\langle\dot{p}_{n}: n \in \omega\right\rangle$ interprets $\left\langle\dot{f}_{0}, \ldots, \dot{f}_{k}\right\rangle$ as $\left\langle\dot{f}_{0}^{*}, \ldots, \dot{f}_{k}^{*}\right\rangle "$.

By induction on $j \in \omega$ we will construct

(i) a sequence of $P_{\beta}$-names of ordinals $\dot{\alpha}_{j} \in N$ such that $\dot{\alpha}_{0}=\alpha, \dot{\alpha}_{j+1}$ is a $P_{\dot{\alpha}_{j}}$-name of an ordinal (recall that $P_{\dot{\alpha}_{j}}=P_{\dot{\alpha}_{0}} * \dot{P}_{\dot{\alpha}_{0}, \dot{\alpha}_{1}} * \ldots * \dot{P}_{\dot{\alpha}_{j-1}, \dot{\alpha}_{j}}$ ) such that $\Vdash_{\dot{\alpha}_{j}} \dot{\alpha}_{j}<\dot{\alpha}_{j+1}<\beta$, and $\Vdash_{\beta} \lim _{j \in \omega} \dot{\alpha}_{j}=\sup (N \cap \beta)$;

(ii) $P_{\dot{\alpha}_{j}}$-names $\left\langle\dot{f}_{0}^{j}, \ldots, \dot{f}_{k+j}^{j}\right\rangle \in N$ for functions and two $P_{\dot{\alpha}_{j}}$-names for decreasing sequences of conditions in $\dot{P}_{\dot{\alpha}_{j}, \beta},\left\langle\dot{p}_{n}^{j}: n \in \omega\right\rangle \in N$ and $\left\langle\dot{p}_{n}^{j *}: n \in\right.$ $\omega\rangle \in N$,

so that the following holds:

$(\ell 1)\left\langle\dot{f}_{0}^{0}, \ldots, \dot{f}_{k}^{0}\right\rangle=\left\langle\dot{f}_{0}^{*}, \ldots, \dot{f}_{k}^{*}\right\rangle,\left\langle\dot{p}_{n}^{0}: n \in \omega\right\rangle=\left\langle\dot{p}_{n}: n \in \omega\right\rangle ;$

$(\ell 2) \Vdash_{\dot{\alpha}_{j}} \dot{p}_{n}^{j *}\left\lceil\dot{\alpha}_{j+1} \Vdash_{\dot{\alpha}_{j}, \dot{\alpha}_{j+1}} \dot{p}_{0}^{j+1} \leq \dot{p}_{n}^{j *} \uparrow\left\langle\dot{\alpha}_{j+1}, \beta\right) ;\right.$

$(\ell 3) \Vdash_{\dot{\alpha}_{j}}$ “ $\left\langle\dot{p}_{n}^{j}: n \in \omega\right\rangle$ interprets $\left\langle\dot{f}_{0}, \ldots, \dot{f}_{k+j}\right\rangle$ as $\left\langle\dot{f}_{0}^{j}, \ldots, \dot{f}_{k+j}^{j}\right\rangle$ ";

$(\ell 4) \Vdash_{\dot{\alpha}_{j}} "\left\langle\dot{p}_{n}^{j *} \mid \dot{\alpha}_{j+1}: n \in \omega\right\rangle$ interprets $\left\langle\dot{f}_{0}^{j+1}, \ldots, \dot{f}_{k+j}^{j+1}\right\rangle$ as $\left\langle\dot{f}_{0}^{j}, \ldots\right.$ $\left.\ldots, \dot{f}_{k+j}^{j}\right\rangle "$

$(\ell 5) \Vdash_{\dot{\alpha}_{j}}$ " $\dot{p}_{0}^{j}$ decides $\dot{f}_{0}\left\lceil j, \ldots, \dot{f}_{k+j}\lceil j " ;\right.$ 


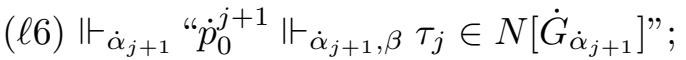

$(\ell 7) \Vdash_{\dot{\alpha}_{j}}(\forall n) \dot{p}_{n}^{j *} \leq p_{n}^{j}$.

Before starting the construction fix an arbitrary strictly increasing sequence $\left\{\alpha_{j}^{\prime}: j \in \omega\right\} \subseteq N$ of ordinals with $\lim _{j} \alpha_{j}^{\prime}=\sup (N \cap \beta)$.

For $j=0$, the conditions $(\ell 1),(\ell 3)$ are given by the assumptions of Lemma 1.8 and $(\ell 5)$ is trivially true. Assume $\dot{\alpha}_{j},\left\langle\dot{f}_{0}^{j}, \ldots, \dot{f}_{k+j}^{j}\right\rangle$ and $\left\langle\dot{p}_{n}^{j}\right.$ : $n \in \omega\rangle$ are defined. Using Lemma 1.9 in $V^{P_{\dot{\alpha}_{j}}}$ with the assumptions forced

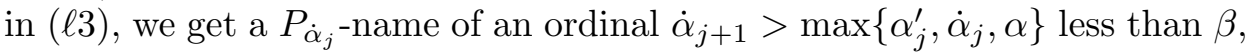
a $P_{\dot{\alpha}_{j}}$-name of a decreasing sequence $\left\langle\dot{p}_{n}^{j *}: n \in \omega\right\rangle, P_{\dot{\alpha}_{j+1}}$-names of functions $\left\langle\dot{f}_{0}^{j+1}, \ldots, \dot{f}_{k+j+1}^{j+1}\right\rangle$ and a $P_{\dot{\alpha}_{j+1}}$-name of a sequence $\left\langle\dot{p}_{n}^{j+1}: n \in \omega\right\rangle$ such that conditions $(\ell 2),(\ell 3)_{j+1}$ and $(\ell 4)-(\ell 7)$ are fulfilled. Also requirements (i), (ii) are satisfied.

Let us make some easy remarks about the names $\dot{\alpha}_{j}$. The name $\dot{\alpha}_{0}$ is in fact the ordinal $\alpha$ and $\dot{\alpha}_{1}$ is a $P_{\alpha}$-name for an ordinal. So if $p \in P_{\beta}$ decides $\dot{\alpha}_{1}$ and $p \Vdash_{\beta}$ " $\dot{\alpha}_{1}=\xi$ " for some $\xi<\beta$ then also $p\left\lceil\alpha\right.$ decides $\dot{\alpha}_{1}, p\left\lceil\xi \in P_{\dot{\alpha}_{1}}\right.$ and $P_{\xi}$ is dense in $P_{\dot{\alpha}_{1}}$ below $p\left\lceil\xi\right.$. In general, if $p \in P_{\beta}$ decides $\dot{\alpha}_{i}$ for $i \leq j$ and forces for them the values $\xi_{i}, i \leq j$, then $p\left\lceil\xi_{j} \in P_{\dot{\alpha}_{j}}\right.$ and $P_{\xi_{j}}$ is dense in $P_{\dot{\alpha}_{j}}$ below $p\left\lceil\xi_{j}\right.$. Moreover,

$$
D_{j}=\left\{p\left\lceil\xi: p \in P_{\beta} \& p \text { decides } \dot{\alpha}_{0}, \ldots, \dot{\alpha}_{j+1} \& p \Vdash_{\beta} \dot{\alpha}_{j}=\xi\right\}\right.
$$

is a dense subset of $P_{\dot{\alpha}_{j}}$ (but not open). On the other hand, $P_{\dot{\alpha}_{j}}$ is not a subset of $P_{\beta}$.

Recall that for $p \in P_{\beta}$,

$$
\operatorname{supp}(p)=\left\{\xi \in \beta: p\left\lceil\xi \Downarrow \xi_{\xi} p(\xi)=\mathbf{1}_{\dot{Q}_{\xi}}\right\} .\right.
$$

By induction on $j \in \omega$ we will construct $N$-generic conditions $r_{j} \in P_{\dot{\alpha}_{j}}$ such that

$(\alpha) r_{j} \in P_{\beta}$ (i.e. $\operatorname{supp}\left(r_{j}\right)$ is countable),

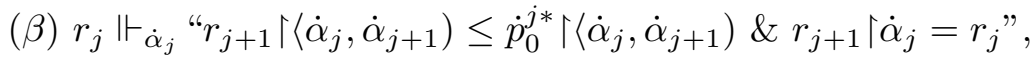

$(\gamma) r_{j} \Vdash_{\dot{\alpha}_{j}}$ " $g$ covers $N\left[\dot{G}_{\dot{\alpha}_{j}}\right]$ ",

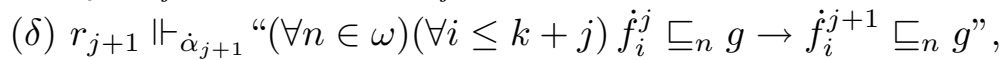

(ع) $r_{j+2}\left\lceil\alpha_{j}^{\prime}=r_{j+1}\left\lceil\alpha_{j}^{\prime}\right.\right.$.

By assumptions of Lemma 1.8, $r_{0}=p$ satisfies $(\alpha)$ and $(\gamma)$. Assume $r_{j}$ is constructed and choose a maximal antichain $A \subseteq D_{j}, A \in N$. Hence every condition $s \in A$ decides $\dot{\alpha}_{j}$ and $\dot{\alpha}_{j+1}$ and so for some ordinals $\alpha_{j, s}, \alpha_{j+1, s}$,

$$
s \Vdash_{\dot{\alpha}_{j}} \dot{\alpha}_{j}=\alpha_{j, s} \& \dot{\alpha}_{j+1}=\alpha_{j+1, s} .
$$

For every $s \in A$ let us fix (in $N) P_{\alpha_{j, s}}$-names $\left\langle\dot{p}_{n}^{j, s}: n \in \omega\right\rangle,\left\langle\dot{f}_{0}^{j, s}, \ldots, \dot{f}_{k+j}^{j, s}\right\rangle$ and $P_{\alpha_{j+1, s}}$-names $\left\langle\dot{p}_{n}^{j+1, s}: n \in \omega\right\rangle,\left\langle\dot{f}_{n}^{j+1, s}: n \in \omega\right\rangle$ such that for every 
$n \in \omega$

$$
\begin{aligned}
& s \Vdash_{\dot{\alpha}_{j}} \dot{p}_{n}^{j, s}=\dot{p}_{n}^{j *} \& \dot{f}_{i}^{j, s}=\dot{f}_{i}^{j}, \quad \text { for } i \leq k+j, \\
& s \Vdash_{\dot{\alpha}_{j}} \Vdash_{\dot{\alpha}_{j}, \dot{\alpha}_{j+1}} \dot{p}_{n}^{j+1, s}=\dot{p}_{n}^{j+1 *} \& \dot{f}_{i}^{j+1, s}=\dot{f}_{i}^{j+1}, \quad \text { for } i \leq k+j+1,
\end{aligned}
$$

and such that analogous conditions to conditions $(\ell 2)-(\ell 7)$ are valid and forced in $P_{\alpha_{j, s}}$ and $P_{\alpha_{j+1, s}}$, respectively. This is possible because $P_{\dot{\alpha}_{j}}, P_{\alpha_{j, s}}$ (and also $P_{\dot{\alpha}_{j+1}}, P_{\alpha_{j+1, s}}$ ) are the same below $s$.

Now when the names $\dot{\alpha}_{j}, \dot{\alpha}_{j+1}$ are fixed by the conditions from $A$, we can use the induction hypothesis, for every $s \in A$ in particular, to get an extension $r_{j+1, s} \in P_{\alpha_{j+1, s}}$ of $r_{j}\left\lceil\alpha_{j, s}\right.$ which is $\left(N, P_{\alpha_{j+1, s}}\right)$-generic and

$(\beta)_{s} r_{j}\left\lceil\alpha_{j, s} \Vdash{ }_{\alpha_{j, s}} r_{j+1, s} \uparrow\left\langle\alpha_{j, s}, \alpha_{j+1, s}\right) \leq \dot{p}_{0}^{j, s} \uparrow\left\langle\alpha_{j, s}, \alpha_{j+1, s}\right) ;\right.$

$(\gamma)_{s} r_{j+1, s} \Vdash_{\alpha_{j+1, s}}$ "g covers $N\left[\dot{G}_{\alpha_{j+1, s}}\right]$ ";

$(\delta)_{s} r_{j+1, s} \Vdash_{\alpha_{j+1, s}}(\forall n \in \omega)(\forall i \leq k+j) \dot{f}_{i}^{j, s} \sqsubseteq_{n} g \rightarrow \dot{f}_{i}^{j+1, s} \sqsubseteq_{n} g$.

Now define $r_{j+1}$ so that for every $s \in A$,

$$
\text { if } s, r_{j} \text { are compatible then } s \Vdash_{\dot{\alpha}_{j}} r_{j+1}=r_{j+1, s} \text {. }
$$

We show that $r_{j+1}$ satisfies $(\alpha)-(\varepsilon)$. Immediately we see that $r_{j+1}$ satisfies $(\beta)$. By genericity of $r_{j}, r_{j}$ meets only countably many elements of $A$ and so $r_{j+1}$ is composed of countably many conditions $r_{j+1, s} \in P_{\dot{\alpha}_{j+1}}$. Therefore the support of $r_{j+1}$ is countable:

$$
\operatorname{supp}\left(r_{j+1}\right) \subseteq \bigcup\left\{\operatorname{supp}\left(r_{j+1, s}\right): s \in A \cap N\right\} .
$$

Since $r_{j} \Vdash_{\dot{\alpha}_{j}}$ " $r_{j+1}\left\lceil\dot{\alpha}_{j}=r_{j} \& r_{j+1}\right\rceil\left\langle\dot{\alpha}_{j}, \dot{\alpha}_{j+1}\right)$ is $N\left[\dot{G}_{\dot{\alpha}_{j}, \dot{\alpha}_{j+1}}\right]$-generic", $r_{j+1}$ is $\left(N, P_{\dot{\alpha}_{j+1}}\right)$-generic and condition $(\alpha)$ is satisfied. Clearly, $(\gamma)$ and $(\delta)$ are also satisfied. The validity of $(\varepsilon)$ follows from the fact that $\Vdash_{\alpha_{j}} \dot{\alpha}_{j+1}>\alpha_{j}^{\prime}$.

Now we define $r=\bigcup_{j \in \omega} r_{j+1}\left\lceil\alpha_{j}^{\prime}\right.$. Since $\operatorname{supp}(r) \subseteq \sup (N \cap \beta), r \in P_{\beta}$. Also, $r\left\lceil\alpha=p\right.$ and $r_{j} \Vdash_{\dot{\alpha}_{j}} r\left\lceil\dot{\alpha}_{j+1}=r_{j+1}\right.$ for $j \in \omega$.

From $(\ell 2)$ and $(\beta)$ it can be seen that

$$
r_{j} \Vdash_{\dot{\alpha}_{j}} r\left\lceil\left\langle\dot{\alpha}_{j}, \beta\right) \leq \dot{p}_{0}^{j *},\right.
$$

and in particular by $(\ell 7), p \Vdash_{\alpha} r \uparrow\langle\alpha, \beta) \leq \dot{p}_{0}$. This proves condition (c) of Lemma 1.8.

Since the condition $r_{j+1}$ is $\left(N, P_{\dot{\alpha}_{j+1}}\right)$-generic,

$$
r_{j+1} \Vdash_{\dot{\alpha}_{j+1}} \text { On } \cap N\left[\dot{G}_{\dot{\alpha}_{j+1}}\right]=\text { On } \cap N
$$

and ( $\ell 6)$ and $(\ell 7)$ give

$$
r_{j+1} \Vdash_{\dot{\alpha}_{j+1}} \dot{p}_{0}^{j+1 *} \Vdash_{\dot{\alpha}_{j+1}, \beta} \tau_{j} \in N .
$$

Hence by $(*)$ we have $r \Vdash_{\beta} \tau \in N$, for every $j \in \omega$. Therefore, $r$ is $\left(N, P_{\beta}\right)$ generic. 
We verify conditions (a) and (b) of Lemma 1.8. By ( $\ell 5)$ and ( $\ell 3)$,

$$
\Vdash_{\dot{\alpha}_{j}} \dot{p}_{0}^{j} \Vdash_{\dot{\alpha}_{j}, \beta} \dot{f}_{i} \uparrow j=\dot{f}_{i}^{j}\lceil j, \quad \text { for } j \geq i-k
$$

and so

By $(\delta)$,

$$
r \Vdash_{\beta}(\forall j \geq i-k) \dot{f}_{i} \uparrow j=\dot{f}_{i}^{j}\lceil j .
$$

and so by $(\gamma)$ for $j_{0}=i-k$,

$$
r \Vdash_{\beta}(\forall j \geq i-k) \dot{f}_{i}^{j} \sqsubseteq_{n} g \rightarrow \dot{f}_{i}^{j+1} \sqsubseteq_{n} g,
$$

$$
r \Vdash_{\beta} \exists n \dot{f}_{i}^{j_{0}} \sqsubseteq_{n} g .
$$

Putting the last three facts together we can find some name $\dot{n}$ for an integer such that $r \Vdash_{\beta}\left(\forall j \geq j_{0}\right) \dot{f}_{i}^{j} \sqsubseteq_{\dot{n}} g$. But the set $\left\{f \in C: f \sqsubseteq_{n} g\right\}$ is closed for every $n$ and $\dot{f}_{i}=\lim _{j \in \omega} \dot{f}_{i}^{j}$ in $V^{P_{\beta}}$, so $r \Vdash_{\beta} \dot{f}_{i} \sqsubseteq \dot{n} g$. This proves (a). A similar reasoning also proves (b).

The proof of Lemma 1.8 and also of Theorem 1.7 is finished.

2. Applications. The main results of this section are proved in [4]. We fill in some details, and arrange material in another way. Theorems 2.10 and 2.15 generalize Lemma 6.15 and Example 6.23 of [4], respectively. As a consequence of the relations in Cichon's diagram we deduce that a forcing notion $P$ preserves the base of the ideal of meager sets if and only if $P$ is $\omega_{\omega} \omega$-bounding and $P$ preserves nonmeager sets. This leads to a new result (Theorem 2.18). The part about preservation of the ${ }^{\omega} \omega$-bounding property is as in [4] and we include it for the sake of completeness. Theorem 2.5 was first proved by S. Shelah [11] for $\omega$-proper forcing notions. Preservation of the base of Lebesgue measure zero sets (Theorem 2.22) is equivalent to preservation of the Sacks property (see [4]) and we have no analogy here for preservation of the Laver property.

We will proceed more or less in this way:

(a) we take into account some 'simple property' of forcing notions which we would like to preserve by countable support iterations;

(b) we find some sequence $\sqsubseteq=\left\langle\Xi_{n}: n \in \omega\right\rangle$ of appropriate relations on ${ }^{\omega} \omega$ (i.e. having all properties stated in the previous section) such that " $\vdash_{P}(\forall f \in V[\dot{G}])(\exists g \in V) f \sqsubseteq g$ " implies "P has the simple property";

(c) we try to prove that the choice of $\sqsubseteq$ in (b) is effective, i.e. if possible, we try to prove that the following conditions are equivalent:

(i) $P$ has the 'simple property';

(ii) $(\forall f \in V[G])(\exists g \in V) f \sqsubseteq g$;

(iii) $P$ almost preserves $\sqsubseteq$;

(iv) $P$ preserves $\sqsubseteq$.

Concerning the relation between (iii) and (iv) we have the following: 
Lemma 2.1. If for all $g$ and all $n$ the set $\{f: f \sqsubseteq g\}$ is relatively open in $C=\operatorname{dom}(\sqsubseteq)$ then $P$ almost preserves $\sqsubseteq$ iff $P$ preserves $\sqsubseteq$.

Proof. $\leftarrow$ is clear.

$\rightarrow$ Assume that $g$ covers $N$ and $\left\langle p_{n}: n \in \omega\right\rangle \in N$ interprets $\left\langle\dot{f}_{0}, \ldots, \dot{f}_{k}\right\rangle \in$ $N$ as $\left\langle f_{0}^{*}, \ldots, f_{k}^{*}\right\rangle \in N$. For $i \leq k$ let $n_{i}=\min \left\{n: f_{i}^{*} \sqsubseteq_{n} g\right\}$. Since the sets $A_{i}=\left\{f: f \sqsubseteq_{n_{i}} g\right\}$ are open in $C$, there is an integer $n^{*}$ such that $C \cap\left[f_{i}^{*}\left\lceil n^{*}\right] \subseteq A_{i}\right.$ for $i \leq k$. As $P$ almost preserves $\sqsubseteq$, we can find an $N$-generic condition $p \leq p_{n^{*}} \leq p_{0}$ such that $p \Vdash$ " $g$ covers $N[\dot{G}]$ ". For each $i, p \Vdash \dot{f}_{i}\left\lceil n^{*}=f_{i}^{*}\left\lceil n^{*}\right.\right.$ and so $p \Vdash \dot{f}_{i} \in C \cap\left[f^{*}\left\lceil n^{*}\right] \subseteq\left\{f: f \sqsubseteq_{n_{i}} g\right\}\right.$. Hence from the minimality of $n_{i}$ we get $p \Vdash(\forall n \in \omega)(\forall i \leq k) f_{i}^{*} \sqsubseteq_{n} g \rightarrow \dot{f}_{i} \sqsubseteq_{n} g$. Therefore $P$ preserves $\sqsubseteq$.

Preservation of ${ }^{\omega} \omega$-bounding property

Definition 2.2. A forcing notion $P$ is called ${ }^{\omega} \omega$-bounding iff $\Vdash_{P}$ $\left(\forall f \in{ }^{\omega} \omega\right)\left(\exists g \in{ }^{\omega} \omega \cap V\right)(\forall n \in \omega) f(n) \leq g(n)$.

There is a natural way to translate this property into the framework of Definition 1.5.

Definition 2.3. For $f, g \in{ }^{\omega} \omega$, we let $f \sqsubseteq_{n}^{b} g$ iff $(\forall k \geq n) f(k) \leq g(k)$, $\sqsubseteq^{b}=\bigcup_{n \in \omega} \sqsubseteq_{n}^{b}$.

Lemma 2.4. Let $P$ be a proper forcing notion. The following conditions are equivalent.

(i) $P$ is ${ }^{\omega} \omega$-bounding;

(ii) $P$ almost preserves $\sqsubseteq^{b}$;

(iii) $P$ preserves $\sqsubseteq^{b}$.

Proof. It is clear that the equivalence (i) $\leftrightarrow$ (ii) is a corollary of the definitions above and Lemma 1.3. The implication (iii) $\rightarrow$ (ii) is trivial.

(ii) $\rightarrow$ (iii). Consider a model $N$ and a sequence $\left\langle p_{n}: n \in \omega\right\rangle$ interpreting $P$-names $\left\langle\dot{f}_{0}, \ldots, \dot{f}_{k}\right\rangle \in N$ as $\left\langle f_{0}^{*}, \ldots, f_{k}^{*}\right\rangle$. Assume that $g \in{ }^{\omega} \omega$ covers $N$.

Since $P$ is ${ }^{\omega} \omega$-bounding, for every $n \in \omega$ we can find $p_{n}^{\prime} \leq p_{n}, p_{n}^{\prime} \in N$, and $f_{i, n} \in{ }^{\omega} \omega \cap N$, for $i \leq k$, such that $p_{n}^{\prime} \Vdash(\forall m) \dot{f}_{i}(m) \leq f_{i, n}(m)$. Define

$$
f_{i}^{\prime}(m)=\max \left\{f_{i, n}(m): n \leq m\right\}, \quad i \leq k, m \in \omega .
$$

Since $g$ covers $N$ we can define

$$
n_{i}=\min \left\{n:(\forall m \geq n) f_{i}^{\prime}(m) \leq g(m)\right\}, \quad i \leq k .
$$

Let $n^{*}=\max \left\{n_{i}: i \leq k\right\}$ and let $p \leq p_{n^{*}}^{\prime}$ be an $N$-generic condition such that $p \Vdash$ " $g$ covers $N[\dot{G}]$ ". Then for every $i \leq k$,

$$
\begin{aligned}
& p \Vdash\left(\forall m \geq n^{*}\right) \dot{f}_{i}(m) \leq f_{i, n^{*}}(m) \leq f_{i}^{\prime}(m) \leq g(m), \quad \text { and } \\
& p \Vdash \dot{f}_{i}\left\lceil n^{*}=f_{i}^{*}\left\lceil n^{*} .\right.\right.
\end{aligned}
$$


This implies $p \Vdash(\forall n) f_{i}^{*} \sqsubseteq_{n}^{b} g \rightarrow \dot{f}_{i} \sqsubseteq_{n}^{b} g$, and so $P$ preserves $\sqsubseteq^{b}$.

We have proved the following theorem.

THEOREM 2.5. The countable support iteration of proper ${ }^{\omega} \omega$-bounding forcing notions is ${ }^{\omega} \omega$-bounding.

Preservation of outer measure. Let $\Omega$ be the set of clopen subsets of ${ }^{\omega} 2$. We let

$$
C^{\mu}=\left\{f \in{ }^{\omega} \Omega:(\forall n \in \omega) \mu(f(n)) \leq 2^{-n}\right\} .
$$

This is a closed set in the product topology of ${ }^{\omega} \Omega$, where $\Omega$ is equipped with the discrete topology. For $f \in C^{\mu}$ the set

$$
A_{f}=\bigcap_{n \in \omega} \bigcup_{k \geq n} f(k)
$$

has measure zero and for every set $H \subseteq{ }^{\omega} 2$ of measure zero there is $f \in C^{\mu}$ such that $H \subseteq A_{f}$.

Definition 2.6. For $f \in C^{\mu}, g \in{ }^{\omega} \omega$ and $n \in \omega$ we let $f \sqsubseteq_{n}^{\mu} g$ iff $(\forall k \geq n) g \notin f(k)$.

Obviously, the set $\left\{f: f \sqsubseteq_{n}^{\mu} g\right\}$ is a closed subset of $C^{\mu}$. Note that $f \sqsubseteq^{\mu} g$ iff $g \notin A_{f}$. Hence we have the following.

Lemma 2.7. $g$ covers $N$ with respect to $\bigsqcup^{\mu}$ iff $g$ is random over $N$.

LEMma $2.8([6])$. For a homogeneous forcing notion $P$ the following conditions are equivalent ( $P$ is homogeneous iff r.o. $P$ is homogeneous):

(i) for every set $A$ of reals, if $\mu^{*}(A)>0$ then $\Vdash_{P} \mu^{*}(A)>0$;

(ii) for every set $A$ of reals and for every real $c>0$, if $\mu^{*}(A)>c$ then $\Vdash_{P} \mu^{*}(A)>c$;

(iii) for every $P$-name $\dot{A}$ of a set of reals and for every real $c>0$, if $p \in P$ and $p \Vdash \mu^{*}(A) \leq c$ then $\mu^{*}\left(\left\{x \in{ }^{\omega} 2: p \Vdash x \in \dot{A}\right\}\right) \leq c$.

Proof. The implications (ii) $\rightarrow$ (iii) and (iii) $\rightarrow$ (i) are easy and do not require homogeneity of $P$.

$($ i $) \rightarrow\left(\right.$ ii). Assume $\mu^{*}(A)>c$ and for some $p \in P, p \Vdash \mu^{*}(A)<c$. So there is a $P$-name $\left\langle\dot{I}_{n}: n \in \omega\right\rangle$ of a sequence of basic clopen sets such that $p \Vdash$ " $A \subseteq \bigcup_{n \in \omega} \dot{I}_{n} \& \sum_{n \in \omega} \mu\left(\dot{I}_{n}\right) \leq c$ ". Choose a decreasing sequence $\left\langle p_{m}: m \in \omega\right\rangle$ of conditions below $p$, a sequence $\left\langle I_{n}: n \in \omega\right\rangle$ of clopen sets and $h \in{ }^{\omega} \omega$ increasing such that

$$
p_{m} \Vdash(\forall n \leq h(m)) \dot{I}_{n}=I_{n} \& \sum_{n>h(m)} \dot{I}_{n}<1 /(m+1) .
$$

Since for every $m \in \omega, \sum_{n \leq h(m)} \mu\left(I_{n}\right) \leq c$ and $h$ is increasing, $\mu^{*}(A-$ $\left.\bigcup_{n \in \omega} I_{n}\right)>0$. Set $B=A-\bigcup_{n \in \omega} I_{n}$. Then $p_{m} \Vdash B \subseteq \bigcup_{n>h(m)} \dot{I}_{n}$. Using 
homogeneity of $P$, we can find $P$-names $\left\langle\dot{I}_{n}^{m}: h(m)<n<\omega\right\rangle$ such that for every $m$,

$$
p \Vdash B \subseteq \bigcup_{n>h(m)} \dot{I}_{n}^{m} \& \sum_{n>h(m)} \mu\left(\dot{I}_{n}^{m}\right)<1 /(m+1) .
$$

But this implies $p \Vdash \mu^{*}(B)=0$, while $\mu^{*}(B)>0$, which contradicts condition (i).

Lemma 2.9. If $P$ almost preserves $\sqsubseteq^{\mu}$ then $P$ satisfies condition (i) of Lemma 2.8.

Proof. Assume $\mu^{*}(A)>0$ and $p \Vdash \mu^{*}(A)=0$. There is a $P$-name $\dot{f}$ such that $p \Vdash " \dot{f} \in C^{\mu} \& A \subseteq A_{\dot{f}}$ ". Let $N \prec H(\chi)$ contain $P, \dot{f}, p$ and let $g \in A$ be random over $N$ (i.e. $g$ covers $N$ ). Then there is $q \leq p, q \Vdash$ " $g$ covers $N[\dot{G}]$ ". Hence $q \Vdash g \notin A_{\dot{f}} \& A \subseteq A_{\dot{f}}$. A contradiction.

Every measure algebra has a stronger property (see the next theorem) than 'preserving $\sqsubseteq^{\mu}$ '. Unfortunately, this property is not preserved by countable support iterations since, at least under $\mathrm{CH}$, it implies c.c.c.

TheOREM 2.10. Let $B=\mathbf{B}(\kappa)$ for some cardinal $\kappa$ be the measure algebra with measure $\nu$ for adding $\kappa$-many random reals. Then

(i) for every $B$-name $\dot{A}$ of a set of reals, if $\Vdash_{B} \mu(\dot{A})=0$ then

$$
\mu\left(\left\{x \in{ }^{\omega} 2:(\exists p \in B) p \Vdash x \in \dot{A}\right\}\right)=0 ;
$$

(ii) $B$ preserves $\sqsubseteq^{\mu}$.

Proof. (i) Let $\dot{r}(\kappa)$ denote the canonical name for a generic sequence from $\kappa$ to 2. There is a Borel set $D \subseteq{ }^{\kappa} 2 \times{ }^{\omega} 2$ which is of $\nu \times \mu$ measure zero and $\vdash_{B} \dot{A}=(D)_{\dot{r}(\kappa)}$. Hence by the Fubini Theorem,

$$
\mu\left(\left\{x \in{ }^{\omega} 2:(\exists p \in B) p \Vdash x \in \dot{A}\right\}\right)=\mu\left(\left\{x \in{ }^{\omega_{2}} 2: \nu\left((D)^{x}\right)>0\right\}\right)=0 .
$$

(ii) It is easy to see that (i) implies condition (i) of Lemma 2.8 , hence by homogeneity of $B, B$ satisfies all conditions (i)-(iii) of Lemma 2.8.

Assume that $N \prec H(\chi), P \in N$ and $\left\langle p_{n}: n \in \omega\right\rangle \in N$ interprets $\left\langle\dot{f}_{0}, \ldots, \dot{f}_{k}\right\rangle \in N$ as $\left\langle f_{0}^{*}, \ldots, f_{k}^{*}\right\rangle$, where $\dot{f}_{i}$ are $B$-names for elements of $C^{\mu}$. Let $g$ be random over $N$. Define $n_{i}=\min \left\{n: f_{i}^{*} \sqsubseteq_{n}^{\mu} g\right\}$ and in $N$,

$$
B_{n}=\left\{z \in{ }^{\omega} 2: p_{n} \Vdash z \in \bigcup_{i \leq k} \bigcup_{m \geq n} \dot{f}_{i}(m)\right\} .
$$

By 2.8(iii), $\mu^{*}\left(B_{n}\right) \leq k \sum_{m \geq n} 2^{-m}=k 2^{-n+1}$ and since $g$ is random over $N$, $g \notin \bigcap_{n \in \omega} B_{n}$. Let $n^{*} \geq \max \left\{n_{0}, \ldots, n_{k}\right\}$ be such that $g \notin B_{n^{*}}$. Then there is $p \leq p_{n^{*}} \leq p_{0}$ such that $p \Vdash(\forall i \leq k) g \notin \bigcup_{m \geq n^{*}} \dot{f}_{i}(m)$. Since $p \leq p_{n^{*}}$, and $p \Vdash \dot{f}_{i}\left\lceil n^{*}=f^{*}\left\lceil n^{*}\right.\right.$ for all $i \leq k$, one can easily verify that

$$
p \Vdash(\forall n \in \omega)(\forall i \leq k) f_{i}^{*} \sqsubseteq_{n}^{\mu} g \rightarrow \dot{f}_{i} \sqsubseteq_{n}^{\mu} g .
$$


It remains to show only that $p$ is $N$-generic and $p \Vdash$ " $g$ is random over $N[\dot{G}]$ ". The former follows from the fact that $B$ has c.c.c. and so every condition in $B$ is $N$-generic. The latter is also forced with value $\mathbf{1}$ in $B$. To see this assume that for some $q \in B$ and some $B$-name $\dot{f}$ we have $q \Vdash " \dot{f} \in C^{\mu} \& g \in \dot{A}_{\dot{f}}$ ". Then $g \in\left\{x: q \Vdash x \in A_{\dot{f}}\right\} \subseteq\left\{x:(\exists q \in B) q \Vdash x \in A_{\dot{f}}\right\} \in N$. This contradicts the fact that $g$ is random over $N$, because by (i) the last set has measure zero.

The next result can also be found in [6] and we do not prove it.

TheOREM 2.11. The Laver forcing preserves $\sqsubseteq^{\mu}$.

Preservation of nonmeager sets and preservation of bases of the ideal of meager sets. Again, let $\Omega$ be the set of clopen subsets of ${ }^{\omega} 2$. We let

$$
C^{c}=\left\{f \in{ }^{\Omega} \Omega:(\forall U \in \Omega) f(U) \subseteq U\right\} .
$$

For $f \in C^{c}$ the set

$$
A_{f}=\bigcup_{U \in \Omega} f(U)
$$

is an open dense subset of ${ }^{\omega} 2$ and for every open dense set $H \subseteq{ }^{\omega} 2$ there is $f \in C^{c}$ such that $A_{f} \subseteq H$.

Definition 2.12. Fix some standard enumeration $\left\{U_{n}: n \in \omega\right\}$ of the set $\Omega$. For $f \in C^{c}, g \in{ }^{\omega} 2$ and $n \in \omega$ we let $f \sqsubseteq_{n}^{c} g$ iff $(\exists k \leq n) g \in f\left(U_{k}\right)$.

Note that $f \sqsubseteq^{c} g$ iff $g \in A_{f}$ and so by the remark before Definition 2.12 we immediately get

Lemma 2.13. $g$ covers $N$ with respect to $\sqsubseteq^{c}$ iff $g$ is a Cohen real over $N$.

Since the set $\left\{f \in C^{c}: f \sqsubseteq_{n}^{c} g\right\}$ is clopen for every $g \in{ }^{\omega} 2$, by Lemma 2.1 we have

Lemma 2.14. For any forcing notion $P, P$ preserves $\sqsubseteq^{c}$ iff $P$ almost preserves $\sqsubseteq^{c}$.

The category analogue of Theorem 2.10 is true.

Theorem 2.15. (i) For every $\mathbf{C}(\kappa)$-name $\dot{A}$ of a set of reals, if $\vdash_{\mathbf{C}(\kappa)}$ " $\dot{A}$ is meager" then the set $\left\{x \in{ }^{\omega} 2:(\exists p \in \mathbf{C}(\kappa)) p \Vdash x \in \dot{A}\right\}$ is meager.

(ii) $\mathbf{C}(\kappa)$ preserves $\bigsqcup^{c}$.

Proof. (i) The proof is analogous to the proof of 2.10(i).

(ii) Let $N \prec H(\chi)$ and let $g$ be a Cohen real over $N$. By Lemma 2.14 and since $\mathbf{C}(\kappa)$ is c.c.c. it is enough to prove that $\vdash_{\mathbf{C}(\kappa)}$ " $g$ is Cohen over $N[\dot{G}]$ ". To the contrary assume that for some $p \in \mathbf{C}(\kappa)$ and for some $\mathbf{C}(\kappa)$-name $\dot{f}$, $p \Vdash g \notin A_{\dot{f}} \& \dot{f} \in C^{c}$. Hence $g \in\left\{x:(\exists q) q \Vdash x \in{ }^{\omega} 2-A_{\dot{f}}\right\} \in N$. By (i) this contradicts the fact that $g$ is Cohen over $N$. 
Lemma 2.16. If $P$ preserves $\sqsubseteq^{c}$ then for every nonmeager set $A \subseteq{ }^{\omega} 2$, $\Vdash_{P}$ "A is nonmeager".

Proof. This is similar to the proof of Lemma 2.9.

It is not known whether the converse of Lemma 2.16 holds. Anyway we have at least the following result (see [3], [8], [12]).

LEMMA 2.17. Let $P$ be a proper forcing notion. The following conditions are equivalent:

(i) $P$ is ${ }^{\omega} \omega$-bounding and preserves $\sqsubseteq^{c}$;

(ii) $P$ is ${ }^{\omega} \omega$-bounding and preserves nonmeager sets;

(iii) every meager set in the generic extension can be covered by a Borel meager set coded in the ground model.

Proof. (i) $\rightarrow$ (ii) follows from Lemma 2.16.

(ii) $\rightarrow$ (iii). Let $A \in V[G]$ be a meager subset of ${ }^{\omega_{2}} 2$. We may assume without loss of generality that $A$ is closed under finite changes of its elements. Since ${ }^{\omega} 2 \cap V$ is nonmeager, there is $x \in{ }^{\omega} 2 \cap V-A$. Let $\left\{x_{n}: n \in \omega\right\} \in V$ be an enumeration of the set $X=\left\{y \in{ }^{\omega} 2 \cap V:(\exists n)(\forall m \geq n) x(m)=x(n)\right\}$. Since $A$ is invariant under rational translations the set $X$ is disjoint from $A$.

The set $A$ is the union of countably many nowhere dense sets $A_{n}$. For every $n \in \omega$, define in $V[G]$ a function $f_{n} \in{ }^{\omega} \omega$ by

$$
f_{n}(k)=\min \left\{m:\left[x_{k}\lceil m] \cap A_{n}=\emptyset\right\} .\right.
$$

By the ${ }^{\omega} \omega$-bounding property of $P$ there is a function $f \in{ }^{\omega} \omega \cap V$ which eventually dominates all $f_{n}$. Then the set

$$
B={ }^{\omega} 2-\bigcap_{m} \bigcup_{k>m}\left[x_{k} \uparrow f(k)\right]
$$

is meager coded in $V$. Let $n \in \omega$. There is $m$ such that $(\forall k>m) f(k) \geq$ $f_{n}(k)$. Hence

$$
A_{n} \subseteq \omega_{2}-\bigcup_{k>m}\left[x_{k}\left\lceil f_{n}(k)\right] \subseteq{ }^{\omega} 2-\bigcup_{k>m}\left[x_{k} \uparrow f(k)\right] \subseteq B\right.
$$

and so also $A \subseteq B$.

(iii) $\rightarrow$ (i). Let $\mathcal{M}$ denote the ideal of meager sets. There are mappings (see [3])

$$
\varphi:{ }^{\omega} \omega \rightarrow \mathcal{M}, \quad \psi: \mathcal{M} \rightarrow{ }^{\omega} \omega
$$

such that $\varphi(f) \subseteq A \rightarrow f \leq^{*} \psi(A)$ and $\varphi, \psi$ are absolute (the definition of $\psi$ depends just on Borel codes of $F_{\sigma}$-sets). Hence $\left\{\psi(A): A \in \mathcal{M}\right.$ is $F_{\sigma}$ coded in $V\}$ is cofinal in ${ }^{\omega} \omega \cap V[G]$. This proves that $P$ is ${ }^{\omega} \omega$-bounding. It remains to show that $P$ almost preserves $\sqsubseteq^{c}$. So let $N \prec H(\chi)$ be a countable elementary substructure and $g$ be a Cohen real over $N, p, P \in N$. 
Let $q \leq p$ be an $N$-generic condition. Then by (iii) and by genericity of $q$ we have

$$
q \Vdash(\forall A \in N[\dot{G}] \cap \mathcal{M})(\exists B \in N \cap \mathcal{M}) A \subseteq B \& g \notin B .
$$

So $q \Vdash$ " $g$ is a Cohen over $N[\dot{G}]$ ".

If $P$ has property (iii) of 2.17 we say that $P$ preserves the base of the ideal of meager sets. Since condition (i) of Lemma 2.17 is preserved by countable support iterations we have the following.

THEOREM 2.18. The iteration of proper forcing notions preserving the base of the ideal of meager sets, preserves the base of the ideal of meager sets.

EXAMPLES 2.19. The following forcing notions preserve the base of the ideal of meager sets (see [10], [7]):

(1) generalized Cohen forcing with support in the dual ideal to a $P$-point,

(2) every forcing notion preserving bases of the ideal of Lebesgue measure zero sets, or equivalently, all forcing notions with the Sacks property (e.g. Silver forcing, Sacks forcing, generalized Cohen forcing with support in a selective ideal).

Preservation of the base of the ideal of Lebesgue measure zero sets. We will work with convergent series. The following result justifies that. Essential ideas of its proof can be found in [1], [9], [3].

TheOREM 2.20. Let $N \subseteq M$ be transitive models of ZFC. The following are equivalent:

(i) every measure zero set in $M$ is covered by a Borel measure zero set in $N$;

(ii) every convergent series of positive reals in $M$ is dominated by a convergent series from $N$;

(iii) for every function $f \in{ }^{\omega} \omega \cap M$ there exists a function $g \in N$ such that $(\forall n \in \omega) f(n) \in g(n) \&|g(n)| \leq n^{2}$.

Let $\mathcal{K}=\left\{f \in{ }^{\omega} \mathbb{Q}_{+}: \sum_{n \in \omega} f(n)<1\right\}$. We say that $P$ is $\mathcal{K}$-bounding if $\Vdash_{P}(\forall f \in \mathcal{K})(\exists g \in \mathcal{K} \cap V)(\forall n) f(n) \leq g(n)$. let

Let us recall that $\mathbb{Q}\left(\mathbb{Q}_{+}\right)$is the set of (positive) rational numbers. We

$$
C^{\mathcal{K}}=\left\{f \in{ }^{\omega}\left({ }^{<\omega} \mathbb{Q}_{+}-\{\emptyset\}\right):(\forall n) \sum_{i \in \operatorname{dom}(f(n))} f(n)(i)<2^{-n}\right\} .
$$

For $f \in C^{\mathcal{K}}$ let $\varepsilon_{f}$ be defined by $\varepsilon_{f}=f(0)^{\wedge} f(1)^{\wedge} \ldots$ Clearly for every $g \in \mathcal{K}$ 
there is $f \in C^{\mathcal{K}}$ such that $g=\varepsilon_{f}$. For $f, g \in C^{\mathcal{K}}$ we let

$$
\begin{array}{lll}
f \sqsubseteq_{n}^{\mathcal{K}} g & \text { iff } \quad(\forall m \geq n) \varepsilon_{f}(m) \leq \varepsilon_{g}(m), \quad \text { and } \\
f \sqsubseteq{ }^{\mathcal{K}} g & \text { iff } \quad(\exists n) f \sqsubseteq_{n}^{\mathcal{K}} g .
\end{array}
$$

Obviously $\left\{f: f \sqsubseteq_{n}^{\mathcal{K}} g\right\}$ is a closed subset of $C^{\mathcal{K}}$ and $C^{\mathcal{K}}$ is closed in the product topology of $\omega(<\omega \mathbb{Q})$ where ${ }^{<\omega} \mathbb{Q}$ is endowed with the discrete topology. Note that $g$ covers $N$ with respect to $\sqsubseteq^{\mathcal{K}}$ iff $(\forall f \in \mathcal{K} \cap N)(\exists n)$ $(\forall m \geq n) f(m) \leq \varepsilon_{g}(m)$.

LEMma 2.21. Let $P$ be a proper forcing notion. The following are equivalent:

(i) $P$ is $\mathcal{K}$-bounding;

(ii) $\Vdash_{P}\left(\forall f \in C^{\mathcal{K}}\right)\left(\exists g \in C^{\mathcal{K}} \cap V\right) f \sqsubseteq{ }^{\mathcal{K}} g$;

(iii) $P$ almost preserves $\sqsubseteq^{\mathcal{K}}$;

(iv) $P$ preserves $\sqsubseteq^{\mathcal{K}}$.

P r o of. The equivalence (i) $\leftrightarrow$ (ii) follows from the definitions. By Lemma 1.3 we have (ii) $\leftrightarrow$ (iii). The implication (iv) $\rightarrow$ (iii) is trivial. We prove (iii) $\rightarrow$ (iv).

Let $\left\langle p_{n}: n \in \omega\right\rangle \in N$ interpret $\left\langle\dot{f}_{0}, \ldots, \dot{f}_{k}\right\rangle \in N$ as $\left\langle f_{0}^{*}, \ldots, f_{k}^{*}\right\rangle$, where $\dot{f}_{i}$ are $P$-names for elements of $C^{\mathcal{K}}$. Let $g$ cover $N$.

For $f \in C^{\mathcal{K}}$ define $m(n, f)=\sum_{i<n}|f(i)|$. By (i), for every $n \in \omega$ we can find in $N$ a series $f_{i, n}, i \leq k$, and a condition $p_{n}^{\prime} \leq p_{n}$ such that

$$
p_{n}^{\prime} \Vdash(\forall m) \varepsilon_{\dot{f}_{i}}(m) \leq f_{i, n}(m) \& \sum_{m \in \omega} f_{i, n}(m) \leq 2^{-n}+\sum_{m \in \omega} \varepsilon_{\dot{f}_{i}}(m) .
$$

Since $p_{n}^{\prime}$ decides $\varepsilon_{\dot{f}_{i}}\left\lceil m\left(n, f_{i}^{*}\right)\right.$, we can easily see that for $i \leq k$,

$$
\sum\left\{f_{i, n}(m): m \geq m\left(n, f_{i}^{*}\right)\right\} \leq 2^{-n}+\sum_{m \geq n} 2^{-m}<2^{-n+2} .
$$

Define

$$
f_{i}^{\prime}(m)=\max \left\{f_{i, n}(m): m\left(n, f_{i}^{*}\right) \leq m\right\}, \quad i \leq k, m \in \omega .
$$

Then

$$
\sum_{m \in \omega} f_{i}^{\prime}(m) \leq \sum_{n \in \omega} \sum\left\{f_{i, n}(m): m\left(n, f_{i}^{*}\right) \leq m\right\}<\infty .
$$

Since $g$ covers $N$ and $f_{i}^{\prime} \in N$ we can define the integers

$$
n_{i}=\min \left\{n:(\forall m \geq n) f_{i}^{\prime}(m) \leq \varepsilon_{g}(m)\right\}, \quad i \leq k .
$$

Let $n^{*}=\max \left\{n_{i}: i \leq k\right\}$ and let $p \leq p_{n^{*}}$ be an $N$-generic condition such that $p \Vdash$ " $g$ covers $N[\dot{G}]$ ". Then for every $i \leq k$,

$$
p \Vdash \varepsilon_{\dot{f}_{i}}\left\lceil m\left(n^{*}, f_{i}^{*}\right)=\varepsilon_{f_{i}^{*}}\left\lceil m\left(n^{*}, f_{i}^{*}\right),\right.\right.
$$


and since $m\left(n^{*}, f_{i}^{*}\right) \geq n^{*}$,

$$
p \Vdash\left(\forall m \geq m\left(n^{*}, f_{i}^{*}\right)\right) \varepsilon_{\dot{f}_{i}}(m) \leq f_{i, n^{*}}(m) \leq f_{i}^{\prime}(m) \leq \varepsilon_{g}(m) .
$$

From this we can easily see that

$$
p \Vdash(\forall n) f_{i}^{*} \sqsubseteq_{n}^{\mathcal{K}} g \rightarrow \dot{f}_{i} \sqsubseteq_{n}^{\mathcal{K}} g
$$

and so $P$ preserves $\bigsqcup^{\mathcal{K}}$.

Immediately we have

THEOREM 2.22. The countable support iteration of proper $\mathcal{K}$-bounding forcing notions is $\mathcal{K}$-bounding.

Preservation of non-adding new reals. Obviously, Theorem 1.7 can be understood as a tool for preservation of non-adding of reals of particular properties. For example, Theorem 2.5 is a preservation theorem for non-adding of unbounded reals. Naturally we can ask about non-adding of dominating reals, Cohen reals, random reals, etc. A partial answer to this question can be found in [5]. There a preservation theorem for limit steps of countable support iterations of proper forcing notions is proved. In particular, no random reals (and no dominating reals, respectively) are added at limit steps provided such reals are not added before. Consequently, countable support iterations of $\sigma$-centered forcing notions do not add random reals.

\section{References}

[1] T. Bartoszyński, Additivity of measure implies additivity of category, Trans. Amer. Math. Soc. 281 (1984), 209-213.

[2] T. Bartoszyński and H. Judah, Measure and Category, in preparation.

[3] D. H. Fremlin, Cichoń's diagram, Publ. Math. Univ. Pierre Marie Curie 66, Sém. Initiation Anal., 1983/84, Exp. 5, 13 pp.

[4] M. Goldstern, Tools for your forcing construction, in: Set Theory of the Reals, Conference of Bar-Ilan University, H. Judah (ed.), Israel Math. Conf. Proc. 6, 1992, 307-362.

[5] H. Judah and M. Repický, No random reals in countable support iterations, preprint.

[6] H. Judah and S. Shelah, The Kunen-Miller chart (Lebesgue measure, the Baire property, Laver reals and preservation theorems for forcing), J. Symbolic Logic 55 (1990), 909-927.

[7] A. W. Miller, Some properties of measure and category, Trans. Amer. Math. Soc. 266 (1981), 93-114.

[8] J. Pawlikowski, Why Solovay real produces Cohen real, J. Symbolic Logic 51 (1986), 957-968.

[9] J. Raisonnier and J. Stern, The strength of measurability hypotheses, Israel J. Math. 50 (1985), 337-349.

[10] M. Repický, Properties of measure and category in generalized Cohen's and Silver's forcing, Acta Univ. Carol.-Math. Phys. 28 (1987), 101-115. 
[11] S. Shelah, Proper Forcing, Springer, Berlin, 1984.

[12] J. Truss, Sets having caliber $\aleph_{1}$, in: Logic Colloquium 76, Stud. Logic Found. Math. 87, North-Holland, 1977, 595-612.

MATEMATICKÝ ÚSTAV SAV

JESENNÁ 5

04154 KOŠICE, SLOVAKIA

Received 21 January 1993;

in revised form 21 July 1993 